\title{
Investigation of Gravity-Related Spontaneous Wave Function Collapse for Non-static Gravitational Field
}

\author{
Ali Rajaei ${ }^{1,2} \cdot$ Majid Rahnama ${ }^{1,2} \cdot$ Yousef Bahrampour $^{3}$
}

Received: 22 November 2014/ Accepted: 17 December 2014/Published online: 8 June 2016

(C) The Author(s) 2016. This article is published with open access at Springerlink.com

\begin{abstract}
Diosi and Penrose have suggested a criterion for spontaneous wave function collapse. According to Penrose the profound conflict between the principle of superposition and general covariance entails the existence of reduction of quantum states, e.g. a quantum superposition of two different space-time geometries will collapse onto one of them. In his proposal, collapse time has an inverse relationship with ill-definedness in the gravitational selfenergy, for the static gravitational fields. Anandan obtained the same result using the fluctuations of the connection of gravitational field. We show that in Newtonian limit of the superposition of a static and non-static gravitational field the results of the methods of Anandan and Penrose are different, but the numerical value of the extra term in Anandan's approach, involving angular velocity, is not even considered in a case of practical interest. Then, we investigate the collapse time of the superposition of a static and non-static gravitational field.
\end{abstract}

Keywords Spontaneous wave function collapse $\cdot$ Nonstatic gravitational field $\cdot$ Superposition of space-times

\section{Ali Rajaei}

alirajaei@sci.uk.ac.ir

Majid Rahnama

Majid.Rahnama@mail.uk.ac.ir

Yousef Bahrampour

bahram@uk.ac.ir

1 Faculty of Physics, Shahid Bahonar University, P.O. BOX 76175, Kerman, Iran

2 Afzal Research Institute, Kerman, Iran

3 Department of Mathematics, Shahid Bahonar University, P.O. BOX 76175, Kerman, Iran

\section{Introduction}

Quantum mechanics and general relativity are the two basic theories in physics and the principal problem is to unify these two theories in the sense that they are approximations of a deeper quantum theory of gravity. We expect this theory to solve the measurement problem in quantum theory and the problem of quantizing gravitation. Some physicists believe that quantum mechanics is correct and it is general relativity that needs to be reformed. On the other hand, there are physicists that say these two theories are incompatible. It appears that to achieve the consistent quantum theory of gravity, quantum mechanics and general relativity should both be reformed. In standard quantum mechanics, it is postulated that when the wave function of a quantum system is measured by a macroscopic device, it no longer follows the linear Schrodinger equation, but instantaneously collapses to one of the wave functions that corresponds to definite measurement results. However, this collapse postulate is not satisfactory, as it does not explain why and how the wave function collapses during a measurement. There have been various proposals concerning the origin of wave function collapse. Among them, the most promising and exciting idea is proposed by Penrose. He takes a radical approach to quantum gravity by bringing gravity into the measurement problem (Penrose 1989, 1994, 1996, 1997, 2004). Indeed, the gravity-induced collapse hypothesis can be traced back to Feynman (1995). In his Lectures on Gravitation, Feynman considered the philosophical problems in quantizing macroscopic objects and contemplates on a possible breakdown of quantum theory. He said, "I would like to suggest that it is possible that quantum mechanics fails at large distances and for large objects, it is not inconsistent with what we do know. If this failure of quantum mechanics is connected with 
gravity, we might speculatively expect this to happen for masses such that $\mathrm{GM}^{2} / \mathrm{hc}=1$ of $M$ near $10^{-5} \mathrm{~g}$ ".

In this paper, we review Penrose's gravity-induced collapse and also Anandan's approach, which obtained the same result in another way. In Penrose's and Anandan's approaches, the superposition of two different macroscopic configurations are assumed, e.g. state superposition of a lump of material that moves horizontally and that of one that does not move, and each lump location is accompanied by the static gravitational field produced by the lump in that location. We consider the superposition of a static and a non-static gravitational field and show that the results of Penrose and Anandan's approaches are different, but the numerical value of the extra term in Anandan's approach is not considered in a case of practical interest. Our motivation for using non-static space-times is that, since both the collapse of wave function and non-static metric are time asymmetric, maybe considering non-static space-time is a part of the solution to the measurement problem. Finally, we study collapse time of the superposition of a static and non-static gravitational field.

\section{Gravity-Related Spontaneous Collapse: The Penrose's Proposal}

Consider the superposition of two different macroscopic configurations. For example, state superposition of a lump of material that moves horizontally and that of one that does not move is given by:

$|\Psi\rangle=\frac{1}{\sqrt{2}}(|\varphi\rangle+|\dot{\varphi}\rangle)$

where $\varphi$ and $\dot{\varphi}$ are macroscopically different. The superposed lump plays the role of the Schrodinger's cat. Supposing the gravitational field of the lump and that each lump location separately represents a stationary state and that energy in each case is the same, can we conclude that the quantum superposition of the two lump locations is a stationary state? Penrose using the profound conflict between general covariance principle and superposition principle, asserts that gravity is responsible for an objective reduction of quantum states, accordingly such superposition is not stationary and collapses (Penrose 1996). His argument is as follows. General covariance principle implies that in absence of any spatial inhomogeneity in the background potentials, there is nothing in the intrinsic nature of the lump location that allows us to distinguish it from any other lump location, whereas to sense the quantum superposition of lump locations, those locations must be distinguishable. In other words, to have just a single Schrodinger equation governing the evolution of the superposed quantum system, we have to identify those two space-times and according to the general covariance principle there is no canonical way of asserting which point of one space-time is to be regarded as the same point in another. Penrose considered the approximate identification. Assume that $f$ and $f$ are the acceleration 3-vectors of the free-fall motion in the two space-times ( $f$ and $f$ are gravitational forces per unit test mass). Penrose postulated that at each point the scalar $(\dot{f}-f) \cdot(f-f)$ is a measure of incompatibility of the identification. The total measure of incompatibility at time $t$ is given by:

$E_{G}=\frac{1}{4 \pi G} \int\left(f^{\prime}-f\right) \cdot\left(f^{\prime}-f\right) \mathrm{d}^{3} x$

That

$f=-\nabla \phi$

We have

$E_{G}=\frac{1}{4 \pi G} \int(\nabla \dot{\phi}-\nabla \phi)^{2} \mathrm{~d}^{3} x$

$E_{G}=-\frac{1}{4 \pi G} \int(\dot{\phi}(x)-\phi(x))\left(\nabla^{2} \dot{\phi}(x)-\nabla^{2} \phi(x)\right) \mathrm{d}^{3} x$

Using the Newtonian description, we have gravitational potential function $\phi$ and $\dot{\varphi}$ corresponding to the two mass distributions $\rho$ and $\rho$ in the two lump configurations. By Poisson's formula

$\nabla^{2} \phi(x)=4 \pi G \rho(x)$

and using the integral formula

$\phi(x)=-G \int \frac{\rho(y)}{|x-y|} \mathrm{d}^{3} y$

Thus,

$E_{G}=G \int \frac{(\rho(x)-\rho(x))(\rho(y)-\rho(y))}{|x-y|} \mathrm{d}^{3} x \mathrm{~d}^{3} y$

which is the gravitational self-energy of the difference between the mass distributions that are involved in the quantum superposition of the two lump locations. The quantity $E_{G}$ is the Newton self-energies $E_{11}+E_{22}$ of the two lumps minus twice their interaction energy $E_{12}$, assuming stationary and static mass distribution. Accordingly, Penrose proposed that quantum superposition involving remarkable mass displacement are unstable, with lifetime of the order of $\tau=\hbar / E_{G}$. This result is obtained in a different way by Anandan (1998). He points out that quantum gravitational effects depend on the fluctuation of the gravitational field. In a weak gravitational field for which the linearized approximation is appropriate, the 
gravitational fields of the superposed states may be regarded as perturbations of a background Minkowski spacetime. The fluctuation of the connection $\Delta \Gamma$ is given by

$$
(\Delta \Gamma)^{2}=\sum \int\left\langle\Psi\left|\left(\hat{\Gamma}_{\mu \nu}^{\rho}-\left\langle\Psi\left|\hat{\Gamma}_{\mu \nu}^{\rho}\right| \Psi\right\rangle\right)^{2}\right| \Psi\right\rangle \mathrm{d}^{3} x
$$

Consider the quantum superposition of the two lump locations as in "Eq. (1)". In Newton-Cartan limit, assuming static and stationary field, the only non-zero connection coefficients are

$\Gamma_{00}^{i}=\frac{\partial \varphi}{\partial x^{i}}$

Anandan proposed that incompatibility of the identification between two space-times is given by

$E_{G}=\frac{1}{4 \pi G}(\Delta \Gamma)^{2}$

For superposition of two gravitational fields "Eq. (1)", where $|\varphi\rangle$ and $|\dot{\varphi}\rangle$ are quantum states of the two lump locations, we have

$\hat{\Gamma}_{\mu \nu}^{\rho}|\varphi\rangle=\Gamma_{\mu \nu}^{\rho}|\varphi\rangle$

$\hat{\Gamma}_{\mu \nu}^{\rho}|\hat{\varphi}\rangle=\dot{\Gamma}_{\mu \nu}^{\rho}|\hat{\varphi}\rangle$

Accordingly, incompatibility of the identification between two space-times is given by

$$
\begin{aligned}
E_{G} & =\frac{1}{4 \pi G} \sum_{\rho \mu \nu} \int\left\{\frac{1}{4}\left(\Gamma_{\mu \nu}^{\rho}\right)^{2}+\frac{1}{4}\left(\dot{\Gamma}_{\mu \nu}^{\rho}\right)^{2}-\frac{1}{2}\left(\Gamma_{\mu \nu}^{\rho}\right)\left(\dot{\Gamma}_{\mu \nu}^{\rho}\right)\right\} \mathrm{d}^{3} x \\
& =\frac{1}{4 \pi G} \int(\nabla \dot{\phi}-\nabla \phi)^{2} \mathrm{~d}^{3} x
\end{aligned}
$$

This is the same result obtained by Penrose "Eq. (2)".

\section{Gravity-Related Spontaneous Collapse with Non-static Gravitational Field}

Penrose in his scheme assumes that each lump location is accompanied by the static gravitational field produced by the lump in that location. One way to generalize this is to obtain the collapse time for the non-static gravitational field (Anandan 1998; Christian 2001). At least theoretically, we can construct the quantum superposition of a static and a non-static gravitational field. Our motivation for using non-static space-time is different from that in reference (Christian 2001). The linear dynamics of quantum mechanics ( $U$-process) seem to conflict with the postulate that during measurement a non-linear collapse of the wave packet ( $R$-process) occurs. The $R$-process is time asymmetric. In an effort to study $R$-process by the geometric approach, we consider the non-static space-time, because this space-time is time asymmetric. Maybe considering non-static space-times is a part of the solution to the measurement problem. This is only a crude proposal. So far, there is no geometric method for studying the measurement process in quantum theory.

Among the known solutions of Einstein's field equations, the only known solution which is appropriate to describe space-times for non-static gravitational field, and has a genuinely Newton-Cartan limit, is the NUT spacetime (Ehlers 1997). The metric of the NUT space-time can be written as

$\mathrm{d} s^{2}=-V\left(\mathrm{~d} t+4 a \sin ^{2} \frac{\theta}{2} \mathrm{~d} \varphi\right)^{2}+V^{-1} \mathrm{~d} r^{2}+\left(r^{2}+a^{2}\right) \mathrm{d} \sigma^{2}$

With

$V=1-2\left(m r+a^{2}\right) /\left(r^{2}+a^{2}\right)$

where $m$ and $a$ are positive constants, $r>0$, and $\mathrm{d} \sigma^{2}$ denotes the standard metric on $S^{2}$ in terms of $(\theta, \varphi)$. The limit model has a flat Galilean metric with $t$ as absolute time and $r, \theta, \varphi$ as Euclidean polar coordinates. In Newton-Cartan limit of the NUT space-time, non-zero connection coefficients are

$\Gamma_{00}^{i}=-g^{i}, \Gamma_{0 i}^{j}=s^{j k} \varepsilon_{i k l} \omega^{l}$

where $s^{j k}$ is a nowhere vanishing, symmetric, 2-contravariant tensor field, the (inverse) spatial metric.

$s^{j k}=\operatorname{diag}(0,1,1,1)$

The limit model has a flat Galilean metric with $t$ as absolute time. In addition, with respect to Euclidean polar coordinates, one finds

$\phi=-\frac{m}{r}-\frac{a^{2}}{r^{2}}$

$\vec{g}=-\vec{\nabla} \phi=\left(-\frac{m}{r^{2}}-\frac{2 a^{2}}{r^{3}}\right) \frac{\partial}{\partial r}$

$\vec{\omega}=-\frac{a}{r^{2}} \frac{\partial}{\partial r}$

where $\phi$ is the Newtonian potential, and the spatial vector fields $\vec{g}(t, \vec{x})$ and $\vec{\omega}(t, \vec{x})$ play the role of a gravitational acceleration and Coriolis angular velocity, respectively. Accordingly, we have a superposition of two different space-time geometries. Thus, there is no way to make the spatial identifications between the two space-times under superposition, so that the free falls agree everywhere throughout the space-time. Thus, similar to what was observed in the static case, we consider some approximate notion of pointwise identification between the two spacetimes under superposition. We compute the error caused by this approximation in non-static case, using the NewtonCartan limit of the NUT space-time by method of 
Anandan. Incompatibility of the identification between two space-times is given by "Eq. (6)". If we had used the method of Anandan, we would have obtained the additional term rather than the method of Penrose, because in this way, there are other components of $\Gamma_{0 i}^{j}$ besides $\Gamma_{00}^{i}$. Consider quantum superposition of the two gravitational fields "Eq. (1)", where one field is static and stationary with connection coefficients $\Gamma_{\mu v}^{\rho}$, and the other is stationary and non-static with connection coefficients $\Gamma_{\mu v}^{\rho}$. By "Eqs. (4)" and "(6)", $E_{G}$ should be of the form

$\frac{1}{4 \pi G} \sum_{\rho \mu v} \int\left\{\frac{1}{4}\left(\Gamma_{\mu v}^{\rho}\right)^{2}+\frac{1}{4}\left(\dot{\Gamma}_{\mu v}^{\rho}\right)^{2}-\frac{1}{2}\left(\Gamma_{\mu v}^{\rho}\right)\left(\dot{\Gamma}_{\mu v}^{\rho}\right)\right\} \mathrm{d}^{3} x$

In Newton-Cartan limit non-zero connection coefficients are

$\Gamma_{00}^{i}=\frac{\partial \varphi}{\partial x^{i}}$

$\dot{\Gamma}_{00}^{i}=\frac{\partial \dot{\varphi}^{\prime}}{\partial x^{i}}$

$\dot{\Gamma}_{0 i}^{j}=s^{j k} \varepsilon_{i k l} \omega^{l}$

By substituting connection coefficients in "Eq. (13)", $E_{G}$ is given by:

$\frac{1}{4 \pi G} \int(\vec{\nabla} \dot{\phi}-\vec{\nabla} \phi)^{2} \mathrm{~d}^{3} x+\frac{1}{2 \pi G} \int \omega^{2} \mathrm{~d}^{3} x$

The second term in "Eq. (14)" is non-zero, and this is different from "Eq. (2)". In Penrose's method, where we compare the geodesics related to any space-time, geodesic equation in Newtonian limit of the geodesic equation is

$\frac{\mathrm{d}^{2} X^{i}}{\mathrm{~d} t^{2}}+\Gamma_{00}^{i} \frac{\mathrm{d} X^{0}}{\mathrm{~d} t} \frac{\mathrm{d} X^{0}}{\mathrm{~d} t}=0$

where in non-static case

$$
\begin{aligned}
\Gamma_{00}^{i} & =-g^{i} \\
& =-\frac{m}{r^{2}}-\frac{2 a^{2}}{r^{3}}
\end{aligned}
$$

But in the method of Anandan connection coefficients $\Gamma_{0 i}^{j}$ and $\Gamma_{00}^{i}$ are present, which yield the additional term. The difference between Penrose's and Anandan's approaches are clear and are to be expected, but the numerical value of the last term in "Eq. (14)", involving angular velocity, is not even considered in a case of practical interest. Accordingly, for all practical purposes Penrose and Anandan's approaches yield the same results.

Now, we obtain the collapse time $\tau$ of the superposition of the two gravitational fields, when one of them is the nonstatic gravitational field for which there are other components of $\Gamma_{0 i}^{j}$ besides $\Gamma_{00}^{i}$. Incompatibility of the identification between two space-times is given by substitution "Eq. (14)" in "Eq. (2)" or "Eq. (6)". For a non-static gravitational field we have (Christian 2001),

$\nabla^{2} \dot{\phi}(x)=4 \pi G \dot{\rho}(x)+2 \omega^{2}(x)$

where $\rho(x)$ and $\omega(x)$ are mass distribution and Coriolis angular velocity of non-static gravitational field, respectively. We can write

$\nabla^{2} \dot{\phi}(x)=4 \pi G\left(\dot{\rho}(x)+\frac{\omega^{2}(x)}{2 \pi G}\right)=4 \pi G \varrho(x)$

Accordingly, $E_{G}$ for non-static case is given by "Eq. (2)" or "Eq. (6)". We have

$E_{G}=-\frac{1}{4 \pi G} \int(\dot{\phi}(x)-\phi(x))\left(\nabla^{2} \dot{\phi}(x)-\nabla^{2} \phi(x)\right) \mathrm{d}^{3} x$

using the integral formula

$\dot{\phi}(x)=-G \int \frac{\varrho}{|x-y|} \mathrm{d}^{3} y$

We obtain

$E_{G}=G \int \frac{(\varrho(x)-\rho(x))(\varrho(y)-\rho(y))}{|x-y|} \mathrm{d}^{3} x \mathrm{~d}^{3} y$

Due to the existence of the additional term caused by Coriolis angular velocity in "Eq. (15)", $E_{G}$ in the non-static case is greater than the static case. Thus, according to $\tau=\hbar / E_{G}$ the collapse time of the superposition of a static and non-static gravitational field is less than the superposition of two static gravitational fields. Since there are no satisfactory measurements of the collapse time in the static case, such experiment seems unlikely in the non-static case.

\section{Conclusions}

We showed that in Newtonian limit, collapse time for the superposition of a static and a non-static gravitational field by the methods of Anandan and Penrose leads to different quantities, but the numerical value of the extra term in Anandan's approach is not considered in the case of practical interest. Since both the collapse of wave function and non-static metric are time asymmetric, we propose that non-static space-time may play a role in the solution of measurement problem and investigate Penrose's gravityinduced collapse for non-static gravitational field. Finally, we study collapse time of the superposition of a static and non-static gravitational field.

Acknowledgments We thank, Pouyan Kiasoroush and Ramin Asadi for useful discussions. 
Open Access This article is distributed under the terms of the Creative Commons Attribution 4.0 International License (http://crea tivecommons.org/licenses/by/4.0/), which permits unrestricted use, distribution, and reproduction in any medium, provided you give appropriate credit to the original author(s) and the source, provide a link to the Creative Commons license, and indicate if changes were made.

\section{References}

Anandan JS (1998) Quantum measurement problem and the gravitational field. In: Huggett SA, Mason LJ, Tod KP, Tsou ST, Woodhouse NMJ (eds) The geometric universe: science, geometry, and the work of roger Penrose. Oxford University Press, Oxford, pp 357-368 (see also gr-qc/9808033)
Christian J (2001) Why the quantum must yield to gravity. In: Callender C, Huggett N (eds) Physics meets philosophy at the Planck Scale. Cambridge University Press, Cambridge, p 305

Ehlers J (1997) Examples of Newtonian Limits of Relativistic Spacetimes. Class Quantum Gravity 14:A119-A126

Feynman R (1995) Feynman lectures on gravitation. In: Hatfield B (ed) Reading. Addison-Wesley, Massachusetts

Penrose R (1989) The emperor's new mind: concerning computers, minds and the laws of physics. Oxford University Press, Oxford

Penrose R (1994) Shadows of the mind: a search for the missing science of consciousness. Oxford University Press, Oxford

Penrose R (1996) On gravity's role in quantum state reduction. Gen Relativ Gravit 28:581-600

Penrose R (1997) The large, the small and the human mind. Cambridge University Press, Cambridge

Penrose R (2004) The road to reality: a complete guide to the laws of the universe. Jonathan Cape, London 